\title{
Comparison of the systemic inflammatory response syndrome between monomicrobial and polymicrobial Pseudomonas aeruginosa nosocomial bloodstream infections
}

\author{
Alexandre R Marra*1,2, Gonzalo ML Bearman², Richard P Wenzel ${ }^{2}$ and \\ Michael B Edmond ${ }^{2}$
}

Address: ${ }^{1}$ Department of Infectious Diseases, Universidade Federal de São Paulo, São Paulo, Brazil and ${ }^{2}$ Department of Internal Medicine, Medical College of Virginia Campus, Virginia Commonwealth University, Richmond, Virginia, USA

Email: Alexandre R Marra* - a.marra@uol.com.br; Gonzalo ML Bearman - gbearman@vcu.edu; Richard P Wenzel - rwenzel@mail2.vcu.edu; Michael B Edmond - medmond@mail2.vcu.edu

* Corresponding author

Published: 31 October 2005

BMC Infectious Diseases 2005, 5:94 doi:10.1186/147/-2334-5-94
Received: I5 June 2005

Accepted: 31 October 2005

This article is available from: http://www.biomedcentral.com/I47I-2334/5/94

(C) 2005 Marra et al; licensee BioMed Central Ltd.

This is an Open Access article distributed under the terms of the Creative Commons Attribution License (http://creativecommons.org/licenses/by/2.0), which permits unrestricted use, distribution, and reproduction in any medium, provided the original work is properly cited.

\begin{abstract}
Background: Some studies of nosocomial bloodstream infection (nBSI) have demonstrated a higher mortality for polymicrobial bacteremia when compared to monomicrobial nBSI. The purpose of this study was to compare differences in systemic inflammatory response and mortality between monomicrobial and polymicrobial nBSI with Pseudomonas aeruginosa.
\end{abstract}

Methods: We performed a historical cohort study on 98 adults with $P$. aeruginosa $(\mathrm{Pa}) \mathrm{nBSI}$. SIRS scores were determined 2 days prior to the first positive blood culture through 14 days afterwards. Monomicrobial $(n=77)$ and polymicrobial BSIs $(n=21)$ were compared.

Results: $78.6 \%$ of BSls were caused by monomicrobial P. aeruginosa infection (MPa) and $21.4 \%$ by polymicrobial $P$. aeruginosa infection (PPa). Median APACHE II score on the day of BSI was 22 for $\mathrm{MPa}$ and 23 for PPa BSIs. Septic shock occurred in $33.3 \%$ of $\mathrm{PPa}$ and in $39.0 \%$ of $\mathrm{MPa}(\mathrm{P}=0.64)$. Progression to septic shock was associated with death more frequently in $\mathrm{PPa}$ (OR 38.5, Cl95 2.9508.5) than MPa (OR 4.5, CI95 I.7-12.I). Maximal SIR (severe sepsis, septic shock or death) was seen on day 0 for PPa BSI vs. day I for MPa. No significant difference was noted in the incidence of organ failure, 7-day or overall mortality between the two groups. Univariate analysis revealed that APACHE II score $\geq 20$ at BSI onset, Charlson weighted comorbidity index $\geq 3$, burn injury and respiratory, cardiovascular, renal and hematologic failure were associated with death, while age, malignant disease, diabetes mellitus, hepatic failure, gastrointestinal complications, inappropriate antimicrobial therapy, infection with imipenem resistant $P$. aeruginosa and polymicrobial nBSI were not. Multivariate analysis revealed that hematologic failure $(\mathrm{p}<0.00 \mathrm{I})$ and APACHE II score $\geq 20$ at BSI onset $(P=0.005)$ independently predicted death.

Conclusion: In this historical cohort study of $n B S I$ with $P$. aeruginosa, the incidence of septic shock and organ failure was high in both groups. Additionally, patients with PPa BSI were not more acutely ill, as judged by APACHE II score prior to blood culture positivity than those with MPa BSI. Using multivariable logistic regression analysis, the development of hematologic failure and APACHE II score $\geq 20$ at BSI onset were independent predictors of death; however, PPa BSI was not. 


\section{Background}

$P$. aeruginosa is an important nosocomial BSI pathogen with a high associated mortality [1]. Although the frequency of Gram-negative sepsis has diminished over the last 20 years, the incidence of polymicrobial nBSI infection has increased [2]. In addition, the mortality associated with nosocomial bloodstream infections, particularly those occurring in the intensive care setting, is greater than that of community acquired BSI [3].

Prior studies of nosocomial bloodstream infection (nBSI) have reported a higher associated mortality with polymicrobial nBSI than with monomicrobial nBSI [4]. These studies compared polymicrobial infections with monomicrobial infections caused by diverse pathogens [5]. As such, few investigators have analyzed the clinical significance of polymicrobial versus monomicrobial BSI with a specific pathogen [6]. In addition, a major difficulty in interpreting prior studies is both the marked heterogeneity of variables and the varied definitions of polymicrobial infection $[4,6,7]$. Little information exists about the systemic inflammatory response in polymicrobial BSI $[4,5]$.

The purpose of this study was to evaluate and compare the inflammatory response, clinical course, and outcomes of monomicrobial and polymicrobial nosocomial BSI due to Pseudomonas aeruginosa.

\section{Methods}

\section{Setting}

The Virginia Commonwealth University Medical Center (VCUMC) is an 820-bed tertiary care facility in Richmond, Virginia. The hospital houses 9 intensive care units (ICUs), including pediatric ICUs and a burn unit. Approximately 30,000 patients are admitted annually.

\section{Study design}

Using the Surveillance and Control of Pathogens of Epidemiological Importance (SCOPE) database of bloodstream infections occurring at 49 U.S. hospitals [1], we identified all patients with a diagnosis of nBSI due to P. aeruginosa at VCUMC from January 1, 1996 through December 31, 2003. Patients were considered to have had BSI due to P. aeruginosa if $\geq 1$ blood culture was positive for this organism. Each patient was only included once at the time of the first BSI. Bacteremia was defined as polymicrobial if microorganisms other than $P$. aeruginosa were recovered from the blood culture within a $24 \mathrm{~h}$ period. If the bloodstream isolate was a potential skin contaminant (e.g., diphtheroids, Propionibacterium spp, Bacillus spp, coagulase-negative staphylococci, or micrococci), the presence of an intravascular catheter and the initiation of targeted antimicrobial therapy were required for the diagnosis, as well as at least 1 of the following findings: temperature of $>38.0^{\circ} \mathrm{C}$ or $<36.0^{\circ} \mathrm{C}$, chills, and or systolic blood pressure of $<90 \mathrm{mmHg}$. Clinical data were concurrently collected by infection control practitioners using a standardized case report form. The data collected routinely included age, gender, location of the patient (ward vs. ICU), clinical service, duration of hospitalization prior to onset of BSI, predisposing clinical conditions, and bloodstream pathogen. Predisposing clinical conditions were required to be present prior to BSI and included neutropenia (defined as an absolute neutrophil count $<500 /$ $\mu \mathrm{l})$, peritoneal or hemodialysis, and central venous catheters. Sources of secondary BSI were identified by cultures obtained from distant sites that yielded the same pathogen. Adverse outcomes that occurred during the hospital stay were recorded. These included organ failure and mortality (7-day and overall hospital). The clinical condition of each patient was classified daily according to systemic inflammatory response syndrome (SIRS) criteria [SIRS, sepsis, severe sepsis or septic shock] and APACHE II scores from two days prior to the first positive blood culture through 14 days afterwards [8,9]. The severity of underlying disease for each patient was classified using the Charlson weighted comorbidity index [10]. Patients who had nosocomial BSI due to monomicrobial $\mathrm{P}$. aeruginosa (MPa) were compared to patients who had nosocomial $\mathrm{BSI}$ due to polymicrobial P. aeruginosa (PPa) nBSI.

\section{Definitions}

The patient's physiological conditions prior to the BSI and on the day of BSI were assessed using the APACHE II score [9]. The clinical condition of each patient during the bloodstream infection was classified daily as SIRS, sepsis, severe sepsis or septic shock using criteria previously published by the American College of Chest Physicians / Society of Critical Care Medicine (ACCP/SCCM) [8]. Systemic Inflammatory Response Syndrome (SIRS) was defined as two or more of the following: (a) temperature $>38^{\circ} \mathrm{C}$ or $<36^{\circ} \mathrm{C}$, (b) heart rate $>90$ beats per minute, (c) respiratory rate $>20$ breaths per minute or a $\mathrm{PaCO}_{2}<32 \mathrm{mmHg}$, or (d) white blood cell count $>12 \times 10^{9} / \mathrm{L}$ or $<4 \times 10^{9} / \mathrm{L}$ or the presence of more than $10 \%$ immature neutrophils.

Sepsis was defined as SIRS associated with P. aeruginosa isolated from at least one blood culture. Severe sepsis was associated with organ dysfunction, hypotension or systemic manifestations of hypoperfusion. Septic shock was defined as sepsis associated with hypotension unresponsive to intravenous fluid challenge or the need for a vasopressor agent. The presence of organ system failure at the time of BSI and during the clinical course was assessed using the criteria described by Fagon [11]. Nosocomial infection and sources of infection were defined according to Centers for Disease Control and Prevention (CDC) criteria [12]. Adequate empiric antimicrobial treatment was defined as therapy administered within 24 hours after 
Table I: Patient characteristics and outcomes, stratified by polymicrobial infection.

\begin{tabular}{|c|c|c|c|c|c|}
\hline \multirow[t]{2}{*}{ VARIABLES } & \multicolumn{2}{|c|}{ Polymicrobial $(n=2 I)$} & \multicolumn{2}{|c|}{ Monomicrobial $(n=77)$} & \multirow[t]{2}{*}{$\mathbf{P}$} \\
\hline & $\mathrm{N}$ & $\%$ & $\mathrm{~N}$ & $\%$ & \\
\hline \multicolumn{6}{|l|}{ Demographic characteristics } \\
\hline Age $>60$ years & 7 & 33.3 & 29 & 37.7 & 0.71 \\
\hline Male gender & 17 & 81.0 & 46 & 59.7 & 0.72 \\
\hline Mean LOS prior to nBSI (days) \pm SD (range) & $26 \pm 28.6(5-121)$ & - & $33 \pm 44.7(2-323)$ & - & 0.38 \\
\hline Mean hospital stay (days) \pm SD (range) & $46.9 \pm 32.0(9-128)$ & - & $69.0 \pm 75.4(5-415)$ & - & 0.05 \\
\hline ICU stay & 19 & 90.5 & 63 & 81.8 & 0.51 \\
\hline \multicolumn{6}{|l|}{ Underlying conditions } \\
\hline Charlson score $\geq 3$ & 4 & 19.0 & 23 & 29.9 & 0.32 \\
\hline Burn injury & 9 & 42.9 & 8 & 10.4 & 0.002 \\
\hline Diabetes mellitus & 5 & 23.8 & 17 & 22.1 & 1.0 \\
\hline Neoplasia & 1 & 4.8 & 19 & 24.7 & 0.064 \\
\hline Neutropenia & 0 & - & 10 & 13.0 & 0.11 \\
\hline Gastrointestinal diseases & 4 & 19.0 & 16 & 20.8 & 1.0 \\
\hline \multicolumn{6}{|l|}{ Therapeutics } \\
\hline Mechanical ventilation & 15 & 71.4 & 47 & 61.0 & 0.38 \\
\hline Central venous line & 19 & 90.5 & 64 & 83.1 & 0.51 \\
\hline Hemodialysis & 3 & 14.3 & 12 & 15.6 & 1.0 \\
\hline TPN & 3 & 14.3 & 21 & 27.3 & 0.22 \\
\hline Transfusion & 8 & 38.1 & 20 & 26.0 & 0.28 \\
\hline Prior antibiotics & 18 & 85.7 & 65 & 84.4 & 0.88 \\
\hline \multicolumn{6}{|l|}{ Conditions related to the clinical course } \\
\hline APACHE II score $\geq 20$ at $B S I$ onset & 15 & 71.4 & 50 & 64.9 & 0.58 \\
\hline Mean time to appropriate antimicrobial therapy (days) & 3.40 & - & 1.7 & - & 0.029 \\
\hline Inadequate antibiotic therapy & 18 & 85.7 & 37 & 48.1 & 0.002 \\
\hline Imipenem resistant $P$. aeruginosa & 6 & 28.6 & 20 & 26.0 & 0.81 \\
\hline \multicolumn{6}{|l|}{ Outcomes } \\
\hline Respiratory failure & 15 & 71.4 & 58 & 75.3 & 0.72 \\
\hline Cardiovascular failure & 7 & 33.3 & 30 & 39.0 & 0.64 \\
\hline Renal failure & 11 & 52.4 & 28 & 36.4 & 0.18 \\
\hline Hematologic failure & 6 & 28.6 & 26 & 33.8 & 0.65 \\
\hline Hepatic failure & 2 & 9.5 & 10 & 13.0 & 1.0 \\
\hline 7-day mortality & 6 & 28.6 & 16 & 20.8 & 0.56 \\
\hline Overall mortality & 8 & 38.1 & 37 & 48.1 & 0.42 \\
\hline
\end{tabular}

blood culture samples were obtained that included the administration of any antimicrobial agent to which the $P$. aeruginosa and the other co-pathogens were susceptible [13], except when a susceptible aminoglycoside was used in conjunction with another antimicrobial to which the organisms were resistant or when a susceptible aminoglycoside was used alone.

\section{Microbiological methods}

Blood cultures (each consisting of aerobic and anaerobic bottles) were processed at the VCUMC clinical laboratory using the BACTEC ${ }^{\circledR} 9240$ blood culture system (Becton Dickinson, Sparks MD).

\section{Statistical analysis}

For continuous variables, mean values were compared using two sample t-tests for independent samples. Differences in proportions were compared using a Chi-square test or Fisher's exact test when appropriate. Mean values were reported \pm 1 SD. All tests of significance are twotailed. When collinearity was identified, the variable with the greatest measure of association was included in the multivariate analysis. Odds ratios were calculated for all variables. Ninety five percent confidence intervals were calculated for all odd ratios. Variables found to be significant in univariate analysis were then entered into a multivariate model. Alpha was set at 0.05. All statistical 
Table 2: Characteristics of 26 co-pathogens isolated in 21 cases of polymicrobial $P$. aeruginosa BSI.

\begin{tabular}{|c|c|c|}
\hline \multirow[t]{2}{*}{ Microorganisms } & \multicolumn{2}{|c|}{ Polymicrobial BSI cases $(n=2 I)$} \\
\hline & $\mathrm{N}$ & $\%$ \\
\hline \multicolumn{3}{|c|}{ Number of agents (associated with $P$. aeruginosaBSI) } \\
\hline 1 & 17 & 80.9 \\
\hline 2 & 3 & 14.3 \\
\hline 3 & I & 4.8 \\
\hline \multicolumn{3}{|l|}{ Agents $(n=26)$} \\
\hline CNS & 4 & 15.4 \\
\hline Staphylococcus aureus* & 3 & 11.5 \\
\hline Enterococcus faecalis & I & 3.8 \\
\hline Enterococcus faecium** & 2 & 7.7 \\
\hline Streptococcus pneumoniae & I & 3.8 \\
\hline Acinetobacter baumannii & 4 & 15.4 \\
\hline Burkholderia cepacia & 2 & 7.7 \\
\hline Enterobacter cloacae & I & 3.8 \\
\hline Klebsiella pneumoniae & 3 & 11.5 \\
\hline Klebsiella oxytoca & I & 3.8 \\
\hline Serratia marcescens & I & 3.8 \\
\hline Candida albicans & 3 & 11.5 \\
\hline
\end{tabular}

CNS = coagulase-negative staphylococci

*Two methicillin-resistant S. aureus

*** One vancomycin-resistant $E$. faecium

analyses were done using the Statistical Package for the Social Sciences software (SPSS, Chicago, IL, USA).

\section{Results}

\section{Study population and patient characteristics}

A total of 160 nosocomial P. aeruginosa BSIs were identified at VCUMC during the eight-year study period. Of these, 19 clinically significant episodes of BSI (11.9\%) were identified in pediatric patients ( $<18$ years of age), 92 episodes were monomicrobial, and 49 episodes were polymicrobial BSI. Fifteen monomicrobial and 12 polymicrobial BSIs had incomplete medical records. In 16 cases co-pathogens were recovered from the blood culture $>24$ hours after the isolation of Pseudomonas aeruginosa. The remaining 77 monomicrobial and 21 polymicrobial BSIs caused by P. aeruginosa were included in the analysis.

Proportions and means for the different variables in the two groups are listed in Table 1 . There were no significant differences in age or gender between the two groups ( $\mathrm{p}=$ 0.71 and $p=0.72$, respectively). Burn injuries were more commonly seen in cases of polymicrobial $P$. aeruginosa BSI vs. monomicrobial cases $(42.9 \%$ vs. $10.4 \%, \mathrm{p}=$ $0.002)$. Underlying malignancy was more commonly seen in the monomicrobial $P$. aeruginosa BSI cohort $(24.7 \%$ vs. $4.8 \%, \mathrm{p}=0.064)$. No statistically significant differences were observed in the proportion of patients with diabetes mellitus or gastrointestinal complications between the two BSI groups. No difference was also observed in Charl- son scores $\geq 3$ between the two groups (19.0\% for PPa vs. $29.9 \%$ for $\mathrm{MPa}, \mathrm{p}=0.32)$. Although the majority of patients acquired $P$. aeruginosa BSI in the intensive care unit setting, no statistically significant differences were noticed between the two comparison groups $(90.5 \%$ for PPa vs. $81.8 \%$ for $\mathrm{MPa}, \mathrm{p}=0.51)$. A central venous catheter was present in more than three-quarters of both groups (90.5\% in PPa vs. 83.1\% in MPa, p = 0.51). Twenty-seven percent of the MPa BSI patients received total parental nutrition compared to $14.3 \%$ of PPa BSI patients ( $\mathrm{p}=$ $0.22)$. A greater proportion of PPa BSI patients $(38.1 \%)$ received blood transfusions compared with $26.0 \%$ of $\mathrm{MPa}$ BSI patients; however, this was not statistically significant ( $p=0.28)$. More than half of patients in both groups needed ventilatory support $(71.4 \%$ in PPa vs. $61.0 \%$ in $\mathrm{MPa}, \mathrm{p}=0.38$ ) prior to the onset of BSI. There was no difference in the use of any class of antimicrobials prescribed prior to Pseudomonas BSI between the two groups $(85.7 \%$ in $\mathrm{PPa}$ vs. $84.4 \%$ in $\mathrm{MPa}, \mathrm{p}=0.88$ ).

The mean interval between hospitalization and the onset of BSI did not differ between the monomicrobial and polymicrobial groups $(26 \pm 28.6$ days vs. $33 \pm 44.7$ days, $\mathrm{p}=$ $0.38)$. However, the overall hospital mean length of stay was significantly longer for MPa BSI $(69.0 \pm 75.4$ vs. 46.9 \pm 32.0 days, $\mathrm{p}=0.05$ ).

\section{Microbiological features}

$78.6 \%$ of BSIs were caused by monomicrobial P. aeruginosa infection (MPa) and $21.4 \%$ by polymicrobial P. aer- 

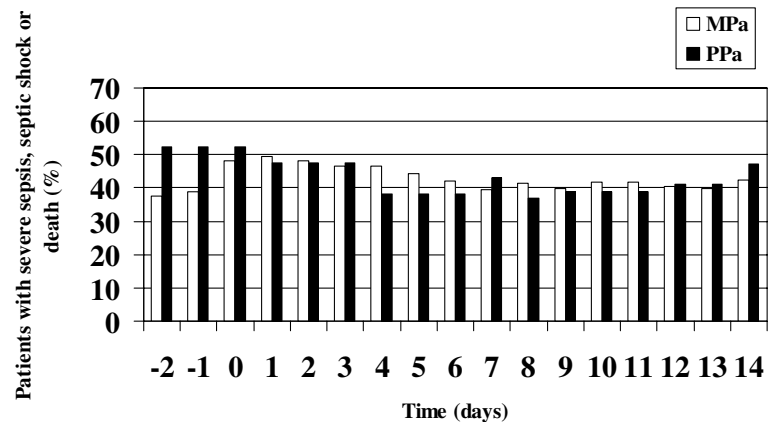

\section{Figure I}

Systemic inflammatory response over time in patients with $P$. aeruginosa $\mathrm{nBSI}$ stratified by polymicrobial infection.

uginosa infection ( $\mathrm{PPa}$ ). The most frequent pathogens associated with polymicrobial P. aeruginosa BSI were coagulase-negative staphylococci (16.6\%), Acinetobacter baumannii (16.6\%), Staphylococcus aureus (12.5\%) and Candida albicans (12.5\%) (Table 2). Polymicrobial infection with more than 2 organisms was seen in $19.1 \%$ of PPa BSI cases. No statistically significant differences were observed in the proportion of imipenem resistance in P. aeruginosa isolates between the two groups $(28.6 \%$ for PPa vs. 26.0\% for $\mathrm{MPa}, \mathrm{p}=0.81$ ).

\section{Clinical course}

Septic shock occurred in 33.3\% of PPa and in 39.0\% of $\mathrm{MPa}(\mathrm{p}=0.64)$. Progression to septic shock was associated with death more frequently in PPa (OR 38.5, CI95 2.9508.5) than MPa (OR 4.5, CI95 1.7-12.1). Maximal SIR (severe sepsis, septic shock or death) was seen on day 0 for PPa BSI vs. day 1 for MPa (Figure 1). Median APACHE II scores on the day of BSI were 23 in the PPa group and 22 in the $\mathrm{MPa}$ group. There was also no difference in APACHE II scores at 14 days post-BSI diagnosis between the MPa and PPa groups (Figure 2). Appropriate empiric antimicrobials were begun within 24 hours in $59.6 \%$ of $\mathrm{MPa}$ and in $14.3 \%$ of PPa $(\mathrm{p}=0.002)$. In addition the time to adequate therapy was twice as long for patients with PPa infection (3.4 days vs. 1.7 days, $\mathrm{p}=0.029$ ). No significant difference was noted in the incidence of organ failure, 7-day mortality, or overall mortality between the two groups as seen in table 1 .

Univariate analysis revealed that APACHE II score $\geq 20$ at BSI onset, Charlson score, burn injury, and respiratory, cardiovascular, renal and hematologic failure were associated with death (table 3). Age, malignancy, diabetes mellitus, hepatic failure, gastrointestinal complications, inappropriate empiric antimicrobial therapy, infection with imipenem-resistant $P$. aeruginosa, and polymicrobial infection were not significant predictors of mortality on

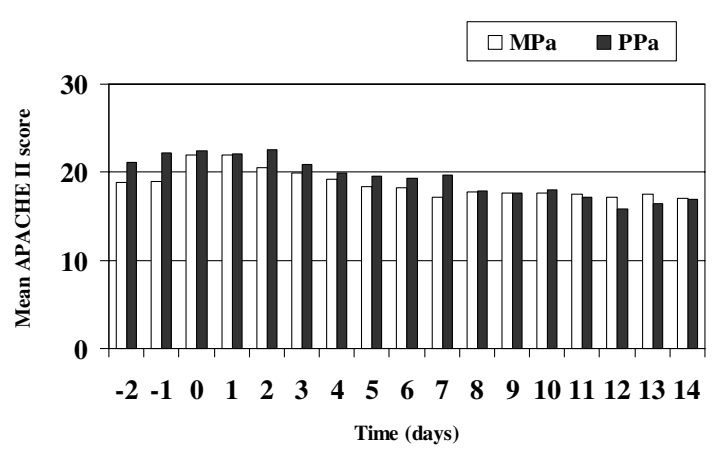

Figure 2

Mean APACHE II scores in patients with P. aeruginosa nBSI stratified by polymicrobial infection.

univariate analysis. Using logistic regression analysis, the following variables were independent predictors for death (table 3): hematologic failure (OR 16.9; CI95 3.9-73.2) and APACHE II score $\geq 20$ at BSI onset (OR 9.7; CI95 1.947.9).

\section{Discussion}

As polymicrobial BSI with $P$. aeruginosa is associated with high mortality, we decided to investigate whether the systemic inflammatory response with polymicrobial $P$. aeruginosa BSI is more intense than the systemic inflammatory response associated with monomicrobial $P$. aeruginosa BSI. During the study period, we found one-fifth of cases were polymicrobial. Since our intention was to analyze the 14-day period after $P$. aeruginosa BSI for the intensity of the systemic inflammatory response, we adopted a more conservative definition of polymicrobial BSI (all organisms isolated in the 24 hour period following the first culture positive for $P$. aeruginosa) rather than the more standard definition (organisms isolated within 48 hours) [14] in order to minimize misclassification of serial monomicrobial infections as polymicrobial infections. It should be noted that the proportion of Pseudomonas BSI cases that were polymicrobial was lower at our hospital than in the SCOPE hospitals overall, where $32.7 \%$ of the 1,256 nosocomial Pseudomonas BSI cases were polymicrobial.

It is also important to note that $19.1 \%$ of the patients with PPa BSI had 2 or 3 co-pathogens associated with $P$. aeruginosa. Coagulase negative staphylococci (CNS) were found in $15.4 \%$ of the PPa BSIs. It is often difficult to determine the pathogenic role of coagulase-negative staphylococci when these organisms are isolated in blood cultures. To avoid this, we utilized more strict criteria for classification of skin flora as pathogens. In addition, in only one of the 
Table 3: Risk factors for in-hospital mortality in patients with $P$. aeruginosa nosocomial bloodstream infection.

\begin{tabular}{|c|c|c|c|c|c|c|c|c|}
\hline \multirow[t]{2}{*}{ VARIABLES } & \multicolumn{2}{|c|}{ Died $(n=45)$} & \multicolumn{2}{|c|}{ Recovered $(n=53)$} & \multicolumn{2}{|c|}{ Univariate analysis } & \multicolumn{2}{|c|}{ Multivariate analysis } \\
\hline & $\mathrm{N}$ & $\%$ & $\mathrm{~N}$ & $\%$ & OR & $\mathrm{Cl} 95 \%$ & OR & $\mathrm{Cl} 95 \%$ \\
\hline Age $>60$ years & 18 & 40.0 & 18 & 34.0 & 1.3 & $0.6-2.9$ & & \\
\hline Burn injury & 12 & 26.7 & 5 & 9.4 & 3.5 & $1.1-10.8$ & 3.2 & $0.6-18.2$ \\
\hline Diabetes mellitus & 10 & 22.2 & 12 & 22.6 & 0.9 & $0.4-2.5$ & & \\
\hline Gastrointestinal complication & 6 & 13.3 & 14 & 26.4 & 0.4 & $0.1-1.2$ & & \\
\hline Neoplasia & 11 & 24.4 & 9 & 17.0 & 1.6 & $0.6-4.2$ & & \\
\hline APACHE II score $\geq 20$ at BSI onset & 41 & 91.1 & 24 & 45.3 & 12.7 & $3.9-39.5$ & 9.7 & $1.9-47.9$ \\
\hline Charlson score $\geq 3$ & 17 & 37.8 & 10 & 18.9 & 2.6 & $1.0-6.5$ & 2.7 & $0.7-10.1$ \\
\hline Respiratory failure & 39 & 86.7 & 34 & 64.2 & 3.6 & $1.3-10.1$ & 1.4 & $0.3-7.2$ \\
\hline Cardiovascular failure & 26 & 57.8 & 11 & 10.8 & 5.2 & $2.1-12.7$ & 2.6 & $0.7-9.3$ \\
\hline Renal failure & 25 & 55.6 & 14 & 26.4 & 3.5 & $|.5-8|$. & 1.3 & $0.4-4.2$ \\
\hline Hematologic failure & 26 & 57.8 & 6 & 11.3 & 10.7 & $3.8-30.2$ & 16.9 & $3.9-73.2$ \\
\hline Hepatic failure & 8 & 17.8 & 4 & 7.5 & 2.6 & $0.7-9.5$ & & \\
\hline Inadequate antibiotic therapy & 26 & 57.8 & 29 & 54.7 & 1.1 & $0.7-1.7$ & & \\
\hline Imipenem resistant $P$. aeruginosa & 16 & 35.6 & 10 & 18.9 & 2.37 & $0.9-5.9$ & & \\
\hline Polymicrobial infection & 8 & 17.8 & 13 & 24.5 & 0.7 & $0.2-1.8$ & & \\
\hline
\end{tabular}

four polymicrobial BSIs in which coagulase-negative staphylococci (CNS) was a co-pathogen was a third organism also found. However, in this particular case, multiple blood cultures yielded both Pseudomonas and CNS, which leads us to believe that both organisms played a pathogenic role.

Acinetobacter baumannii was found in $15.4 \%$ of the polymicrobial cases. A previous study found that Acinetobacter spp. may be a co-pathogen in $P$. aeruginosa polimycrobial BSI [6]. Although both pathogens are nonfermentative Gram-negative bacteria causing nosocomial infections, principally in intensive care units, they show different epidemiologic characteristics [1].

Interestingly, no significant difference was noted between the MPa BSI and PPa BSI cohorts with respect to gastrointestinal complications, such as bowel perforation or gastrointestinal procedures. As such, a gastrointestinal source for the etiology of the polymicrobial BSI is not clearly defined. However, burn injury was more common in PPa BSI ( $p=0.002)$. The loss of the natural cutaneous barrier to infection likely led to microbial colonization and subsequent invasion of the bloodstream [15]. Of note was that malignant conditions were more commonly associated with MPa BSI than PPa BSI.

No difference in APACHE II scores was noted between the two comparison groups during 14 days of follow up. On analysis of severe sepsis, septic shock and death, no statistically significant differences were observed between the $\mathrm{MPa}$ and PPa groups. A previous study by Aliaga et al. showed that patients with polymicrobial infection involving $P$. aeruginosa were worse clinically and developed shock more frequently [6]. However, the severity of underlying diseases chosen by these authors was only evaluated by the McCabe classification. In our study, the Charlson weighted comorbidity index and serial APACHE II scores were used to assess the patients' severity of illness (figure 2).

Our study also demonstrated the difficulty in choosing empiric antimicrobial treatment for polymicrobial infections at the time $P$. aeruginos $a$ was isolated. This difficulty is even greater if organisms such as VRE or Candida are copathogens where it is likely that therapy will be inadequate until all pathogens have been identified [13]. This at least partially explains the difference observed in inadequate empiric treatment in patients with PPa compared to patients with $\mathrm{MPa}(85.7 \%$ vs. $48.1 \%, \mathrm{p}=0.025)$. It is also important to note that there was no difference in imipenem resistance between the PPa and MPa groups $(\mathrm{p}=$ $0.81)$.

By univariate analysis several organ dysfunctions (respiratory, cardiovascular, renal and hematologic) were associated with death, but age, hepatic failure, inappropriate antimicrobial therapy, imipenem resistance and polymicrobial infection were not. The clinical belief that polymicrobial BSI portends a worse prognosis than monomicrobial BSI was not demonstrated in our study. Other studies, however, have associated polymicrobial infection with higher mortality $[4,5]$. Another surprising finding was that the mean length of hospital stay was higher for the monomicrobial group than the polymicrobial group. Thus, our findings suggest that the outcome of $P$. aeruginosa BSI is more closely related to the underlying physiological response to sepsis than it is to polymicrobial infection. However, it must be emphasized that because of the relatively small sample size of our study, a 
difference of at least $28 \%$ between outcome events in the monomicrobial and polymicrobial groups would be necessary to detect a statistically significant difference in SIRS, organ failure or mortality rate. In the SCOPE hospitals overall, the crude mortality of polymicrobial $P$. aeruginosa BSI $(42.4 \%)$ was significantly higher than that seen with monomicrobial $P$. aeruginosa BSI $(34.8 \%),(\mathrm{p}=0.01)$. Thus, the smaller sample size in our study could have led to a type II error in our finding of no significant difference in mortality between mono- and polymicrobial BSI.

In conclusion, in patients with $P$. aeruginosa nBSI, onefifth of cases are polymicrobial, the incidence of septic shock and organ failure is high, patients with PPa BSI are not more severely ill prior to infection than those with MPa BSI, and APACHE II score $\geq 20$ at BSI onset and the development of hematologic failure are independent predictors of death.

\section{Competing interests}

The author(s) declare that they have no competing interests.

\section{Authors' contributions}

ARM participated in the design of the study, collected the data and performed the statistical analysis. GMLB participated in the design of the study and performed the statistical analysis. RPW participated in the design of the study and coordination. MBE conceived of the study, and participated in its design and coordination. All authors read and approved the final manuscript.

\section{Acknowledgements}

This work was supported by CAPES - Coordenação de Aperfeiçoamento de Pessoal de Nível Superior (Brasília, Brazil).

\section{References}

I. Wisplinghoff H, Bischoff T, Tallent SM, Seifert H, Wenzel RP, Edmond MB: Nosocomial bloodstream infections in US hospitals: analysis of 24, I79 cases from a prospective nationwide surveillance study. Clin Infect Dis 2004, 39(3):309-3I7.

2. Martin GS, Mannino DM, Eaton S, Moss M: The epidemiology of sepsis in the United States from 1979 through 2000. New Engl J Med 2003, 348(16): I546-I554.

3. Blot S, Vandewoude K, De Bacquer D, Colardyn F: Nosocomial bacteremia caused by antibiotic-resistant Gram-negative bacteria in critically ill patients: clinical outcome and length of hospitalization. Clin Infect Dis 2000, 34(I 2): 1600-1606.

4. Pittet D, Li N, Wenzel RP: Association of secondary and polymicrobial nosocomial bloodstream infections with higher mortality. Eur J Clin Microbiol Infect Dis 1993, I2(II):813-819.

5. Pittet D, Li N, Woolson RF, Wenzel RP: Microbiological factors influencing the outcome of nosocomial bloodstream infections: a 6-year validated, population-based model. Clin Infect Dis 1997, 24(6): 1068-1078.

6. Aliaga L, Mediavilla JD, Llosá J, Miranda C, Rosa-Fraile M: Clinical significance of polymicrobial versus monomicrobial bacteremia involving Pseudomonas aeruginosa. Eur J Clin Microbiol Infect Dis 2000, I9 (II):87I-874.

7. Laupland KB, Zygun DA, Davies HD, Church DL, Louie TJ, Doig CJ: Population-based assessment of intensive care unit-acquired bloodstream infections in adults: Incidence, risk factors, and associated mortality rate. Crit Care Med 2002, 30(II):2462-2467.

8. American College of Chest Physicians/Society of Critical Care Medicine Consensus Conference: Definitions for sepsis and organ failure and guidelines for the use of innovative therapies in sepsis. Crit Care Med 1992, 20(6):864-874.

9. Knaus WA, Draper EA, Wagner DP, Zimmerman JE: APACHE II: a severity of disease classification system. Crit Care Med 1985, 13(10):818-829.

10. Charlson ME, Pompei P, Ales KL, MacKenzie CR: A new method of classifying prognostic comorbidity in longitudinal studies: development and validation. J Chronic Dis 1987, 40(5):373-383.

II. Fagon JY, Chastre J, Novara A, Medioni P, Gibert C: Characterization of intensive care unit patients using a model based on the presence or absence of organ dysfunctions and/or infection: the ODIN model. Intensive Care Med 1993, I9(3): |37-144.

12. Garner JS, Jarvis WR, Emori TB, Horan TC, Hughes JM: CDC definitions for nosocomial infections. Am J Infect Control 1988, 16(3): $128-140$.

13. Ibrahim EH, Sherman G, Ward S, Fraser VJ, Kollef MH: The influence of inadequate antimicrobial treatment of bloodstream infections on patient outcomes in the ICU setting. Chest 2000, II8(I):9-1I.

14. Weinstein MP, Murphy JR, Reller LB, Lichtenstein KA: The clinical significance of positive blood cultures: a comprehensive analysis of $\mathbf{5 0 0}$ episodes of bacteremia and fungemia in adults. II. Clinical observations, with special reference to factors influencing prognosis. Rev Infect Dis 1983, 5(I):54-70.

15. Mayhall CG: The epidemiology of burn wound infections: then and now. Clin Infect Dis 2003, 37(4):543-550.

\section{Pre-publication history}

The pre-publication history for this paper can be accessed here:

http://www.biomedcentral.com/1471-2334/5/94/prepub 